Recepción: 22/11/2018

Aceptación: 15/11/2018

Publicación: 20/02/2019

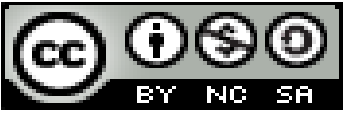

Ciencias de la educación

Artículo de revisión

\title{
Estudio de la aplicabilidad de los conocimientos de Educación Física en el campo laboral turístico: Caso Ecuador
}

\section{Study of the applicability of knowledge of Physical Education in the tourist work field: Case Ecuador}

\section{Estudo da aplicabilidade do conhecimento da Educação Fúsica no campo do turismo: Case Ecuador}

Marco Antonio Toledo-Villacís ${ }^{\mathrm{I}}$ marco.toledo@espoch.edu.ec

Fausto Iván Guapi-Guamán ${ }^{\text {II }}$ fausto.guapi@espoch.edu.ec

Ramiro Vicente Herrera-Jaramillo III ramiro.herrera@espoch.edu.ec

\section{Aurora Azucena Ariza-Velasco IV aurora.ariza@espoch.edu.ec}

Correspondencia: marco.toledo@espoch.edu.ec

\footnotetext{
${ }^{\text {I } M a g i ́ s t e r ~ e n ~ G e r e n c i a ~ d e ~ P r o y e c t o s ~ d e ~ E c o t u r i s m o, ~ D i p l o m a ~ S u p e r i o r ~ e n ~ P r o m o c i o ́ n ~ d e ~ E c o t u r i s m o, ~ I n g e n i e r o ~ E n ~ E c o t u r i s m o, ~}$ Docente de Escuela Superior Politécnica del Chimborazo, Extensión Norte Amazónica, Chimborazo. Ecuador.

II Máster en Investigación en Pedagogía de la Cultura Física y el Deporte, Licenciado en Educación Física y Deporte, Docente de Escuela Superior Politécnica del Chimborazo, Extensión Norte Amazónica, Chimborazo. Ecuador.

III Magíster en Gestión de Proyectos, Ingeniero en Administración y Producción Agropecuaria, Técnico Superior en Administración de Fincas, Docente de Escuela Superior Politécnica del Chimborazo, Extensión Norte Amazónica, Chimborazo. Ecuador.

IV Magíster en Gerencia de Proyectos Educativos y Sociales, Diploma Superior en Diseño de Proyectos, Especialista en Liderazgo y Gerencia, Licenciada en Ciencias de la Educación Profesora de Enseñanza Media en la Especialización de Psicología Educativa y Orientación, Docente de Escuela Superior Politécnica del Chimborazo, Extensión Norte Amazónica, Chimborazo. Ecuador.
} 


\section{Resumen}

La formación profesional que otorgan actualmente las universidades del Ecuador, por exigencia del Consejo de Educación Superior (CES), parte de un estudio de pertinencia que se basa principalmente en las necesidades locales regionales y nacionales. Ese es el punto de partida y de vinculación de la academia con los sectores productivos y sociales del país que justifica la importancia de generar investigación que oriente y garantice la formación integral de los profesionales de turismo. Este trabajo de investigación estudia los conocimientos adquiridos en la asignatura de Educación Física y su aplicabilidad en el campo laboral por parte de los turismólogos en el Ecuador, para ello se hizo un estudio a nivel nacional. La población estuvo constituida por la Red de Profesionales en Turismo del Ecuador REDPROTUR. Los resultados demuestran que los conocimientos adquiridos en la asignatura mencionada, tienen poca aplicabilidad en el campo laboral de los profesionales en turismo y por lo tanto la academia dentro de su competencia debe reestructurar los contenidos básicos y metodologías de enseñanzaaprendizaje que se ejecutan en las carreras de Turismo y orientarlos hacia las verdaderas necesidades que se necesitan en el ámbito turístico nacional en cada una de sus cuatro regiones.

Palabras claves: Ecuador; aplicabilidad; conocimientos; educación física; turística.

\section{Abstract}

The professional training currently granted by the universities of Ecuador, as required by Consejo de Education Superior CES; it is part of the relevance study based mainly on local, regional and national needs. This is the point of departure and linkage of the academy with the productive and social sectors of the country that justifies the importance of generating research that orients and guarantees the integral formation of tourism professionals. This research studies the knowledge acquired in the Physical Education subject and its applicability in the field of work by turismologyts in Ecuador, for which it was done at a national level. The population was constituted by Red de Profesionales en Turismo del Ecuador REDPROTUR. The results show the knowledge acquired in the aforementioned subject, It has little applicability in the professional field of tourism professionals and therefore the academy within its competence must restructure the basic contents and teaching-learning methodologies that are executed in the Tourism careers and guide them towards the real needs that are needed in the national tourism field in each of its four regions. 
Key words: Ecuador; applicability; knowledge; physical education; touristic.

\section{Resumo}

A formação profissional atualmente concedida pelas universidades do Equador, conforme exigido pelo Conselho Superior de Educação do CES, baseia-se em um estudo de relevância baseado principalmente nas necessidades locais, regionais e nacionais. Este é o ponto de partida e articulação da academia com os setores produtivos e sociais do país, o que justifica a importância de gerar pesquisas que orientem e garantam a formação integral dos profissionais do turismo. Esta pesquisa estuda os conhecimentos adquiridos na disciplina de Educação Física e sua aplicabilidade no campo de trabalho por turismologistas no Equador, para isto foi feito um estudo em nível nacional. A população foi constituída pela Rede de Profissionais em Turismo do Equador REDPROTUR. Os resultados demonstram que os conhecimentos adquiridos no referido assunto têm pouca aplicabilidade no campo de trabalho dos profissionais de turismo e, portanto, a academia dentro de sua competência deve reestruturar os conteúdos básicos e as metodologias de ensino-aprendizagem que são implementadas no meio acadêmico. Carreiras de turismo e orientálos para as necessidades reais que são necessárias no campo do turismo nacional em cada uma das suas quatro regiões.

Palavras-chave: equador; aplicabilidade; conhecimento; educação física; turista

\section{Introducción}

La Educación Física es una asignatura a través del cual se imparten conocimientos teóricos y prácticos a los estudiantes, en la mayoría de los países de América Latina, esta materia forma parte de la estructura del sistema educativo y se imparte en el nivel básico, medio y superior; en cada nivel los objetivos y contenidos tributan y persiguen un mismo fin. En la República del Ecuador la Educación Física a nivel superior cumple con lo que estipula la Ley del Deporte, Educación Física y Recreación, en vigencia desde el 10 de agosto de 2010, en la décimo octava disposición general; que plantea: "Educación Física es una disciplina que basa su accionar en la enseñanza y perfeccionamiento de movimientos corporales, busca formar de una manera integral y armónica al ser humano, estimulando positivamente sus capacidades físicas." (Asamblea Nacional, 2008, pág. 24). Sin embargo, en el campo turístico, el profesional no únicamente se debe limitar al aprendizaje de los fundamentos básicos y específicos de los deportes como la 
natación, fútbol, atletismo, básquet etc., si no que requiere además el aprendizaje de habilidades propias al campo de acción laboral que se necesita en turismo.

De acuerdo con la literatura especializada sobre el estudio de la aplicabilidad de la Educación Física en las carreras, existen investigaciones interesantes aplicadas a carreras de ingeniería y licenciatura que destacados autores han abordado la temática entre los que se encuentran Fresneda, Herrera y Álvarez (2013), quienes hacen referencia al desarrollo de las habilidades profesionales específicas de los egresados de la carrera de Medicina Veterinaria; Charchabal, Aguirre y Pérez (2003), abordan la preparación física profesional en los egresados de la carrera de Ingeniería en Minas; cada uno de los aportes realizados por los autores citados anteriormente, coinciden en que la Educación Física es sumamente importante en la formación integral de los estudiantes universitarios a través de la enseñanza y aprendizaje de habilidades propias de la profesión; sin embargo han detectado que la formación en su complejidad no se ajusta enteramente a los fines de sus competencias laborales.

No obstante, al realizar la búsqueda bibliográfica no se ha podido evidenciar la existencia de un estudio de la aplicabilidad de la materia de Educación Física en el campo laboral turístico, por lo que se plantea esta investigación como una opción que aporte con información real dada por los profesionales e involucrados en el turismo en base a su propia experiencia. El técnico en turismo durante su formación desarrolla competencias y habilidades para desenvolverse en el sistema turístico local, provincial, nacional e internacional de acuerdo con sus diferentes manifestaciones entre los que se encuentran los recursos turísticos donde se realizan diferentes actividades físicorecreativas por lo que debe tener un nivel de preparación psicológico, físico, técnico y teórico para brindar los servicios de acuerdo a las necesidades y preservando la integralidad de los recursos naturales y culturales. En este sentido se establece claramente una relación entre la práctica de actividades físico-recreativas dentro de las cuales se encuentra el deporte recreativo y el turismo.

Actualmente la oferta de bienes y servicios están adquiriendo un interés especialmente en el área recreativa y de esparcimiento, estos se caracterizan por ser de gran diversidad, por ejemplo, cabe distinguir prácticas con un perfil puramente recreativo (paseos, comidas campestres, contemplación de la naturaleza, etc.), otras de interés interpretativo-educativo (rutas ecológicas, 
visita a granjas-escuelas, etc.) o aquellas en las que prima la finalidad lúdico-deportiva (Luque, 2003, pág. 133).

Al realizar la revisión del plan de estudios de la carrera de turismo de algunas universidades del país, la observación es la misma, no se desarrolla una formación orientada a las necesidades y habilidades profesionales para desenvolverse en el campo laboral turístico. Los contenidos programáticos y los temas desarrollados que se enseña en la asignatura de Educación Física son en su mayoría, capacidades físicas y elementos técnicos de disciplinas como el fútbol, baloncesto y natación.

A partir de estos antecedentes se plantea la hipótesis de que el programa actual de Educación Física, que se está ofertando y ejecutando en las instituciones de educación superior en el Ecuador, no aportan con el desarrollo de conocimientos, habilidades y destrezas que realmente necesita el profesional en sus actividades turísticas, en este contexto el objetivo principal de este trabajo investigativo, será realizar el estudio de la aplicabilidad de los conocimientos de Educación Física en el campo laboral turístico y el objetivo secundario será proponer en base a los datos obtenidos en la investigación una restructuración de los programas y contenidos de la asignatura mencionada en la educación superior apuntalados y enfocados a la realidad laboral turística.

\section{Importancia del problema}

Las Instituciones de Educación Superior como Institutos, Universidades y Escuelas Politécnicas de la República del Ecuador tienen la misión de formar profesionales competentes; para lo cual los objetivos y habilidades a desarrollar en los estudiantes deben estar explícitos desde el plan de estudios de la carrera y el programa analítico de cada asignatura pues estos constituyen los instrumentos pedagógicos que guían el proceso de enseñanza-aprendizaje del docente. Sin duda las habilidades profesionales se deben desarrollar durante toda la carrera universitaria, donde cada una de las asignaturas del futuro profesional en Turismo deben propiciar al desarrollo de estas, en concordancia con lo que manifiesta Montes de Oca \& Machado, E., (2009) cuyo criterio es que las denominadas habilidades profesionales son desarrolladas fundamentalmente desde disciplinas y asignaturas del ejercicio de la profesión; no obstante, las materias del ciclo básico o 
específico de alguna manera deben tributar también a la formación y desarrollo de dichas habilidades de modo que sea posible desde ellas, crear condiciones para su desarrollo.

En este sentido la Educación Física al ser una asignatura de la unidad básica de la formación del profesional en Turismo debe relacionarse con algunas materias específicas como guianza turística, por ejemplo, o del ejercicio de la profesión siendo esta la base de la formación de los conocimientos que serán aplicados durante el desempeño profesional y de manera específica en el ámbito turístico.

El turismólogo tiene un amplio campo de acción por tanto su desempeño una vez graduado depende en gran medida de la oportunidad y la inclinación que tenga para ejercer su profesión; "La Naturaleza está, y puede seguir jugando un papel preponderante en el desarrollo turístico y es ahí, en el impacto social que presenta esta nueva concepción del turismo relacionado con la actividad física. (Zagalaz, Moreno, \& Chacón, 2001, pág. 278). Ante esta realidad es importante que el futuro turismólogo reciba los contenidos y técnicas que contribuyan a desarrollar habilidades para desempeñarse en su ámbito como por ejemplo el servicio de guianza turística donde está implícito la preparación tanto física como psicológica de las personas para enfrentarse a actividades físico-recreativas en tierra, agua y aire.

Otra de las áreas con que se relaciona el profesional en Turismo es la organización de eventos deportivos partiendo del hecho de la relación existente entre las dos dimensiones sociales; su organización “...conlleva conocer el deporte protagonista, capacidad de gestión y rigor de su práctica, transcurriendo por fases la planificación, programación y el control." (González \& Suárez, 2012, pág. 139). En este sentido le es de gran utilidad al profesional poseer conocimientos relacionados con el proceso de organización y los deportes que en su región se promueva. La relación existente entre turismo y deporte conlleva asumir otros postulados y criterios que no es objetivo del presente artículo; sin embargo, queda plasmado la concepción de que, a través de la asignatura de Educación Física en la educación superior se debe complementar la formación del futuro técnico en turismo.

\section{Metodología}

El segmento objetivo del presente estudio lo constituye la Red de Profesionales de Turismo del Ecuador (REDPROTUR); conformado por ciento setenta y nueve personas profesionales y 
relacionadas en la rama turística, la información fue obtenida directamente mediante una investigación de campo empleando la técnica de encuesta. Se analizaron dos variables: los conocimientos adquiridos en la materia de Educación Física durante los estudios superiores y su aplicabilidad en las actividades turísticas.

Para el efecto se determinó la muestra considerando los datos que se evidencian a continuación:

Fórmula aplicada para el cálculo de la muestra

$$
\begin{aligned}
& \mathrm{Z}^{2} \text {. N. p.q } \\
& \mathrm{n}= \\
& \mathrm{i}^{2}(\mathrm{~N}-1)+\mathrm{Z}^{2} \cdot \mathrm{p} \cdot \mathrm{q}
\end{aligned}
$$

(N) Tamaño del universo: 179 profesionales.

(i) Error: 5\%

(z) Nivel de Confianza 90\% $(1,65)$

(p) Probabilidad de ocurrencia $50 \%$

Tamaño de la muestra 108 profesionales.

El instrumento para la encuesta fue un cuestionario que fue previamente validado por un equipo de profesionales de la carrera de Turismo de la Escuela Superior Politécnica de Chimborazo Extensión Norte Amazónica. La encuesta se la aplicó en línea utilizando la herramienta disponible en google drive con un tipo de muestreo probabilístico - aleatorio simple (muestreo simple al azar), teniendo como marco muestra la lista de socios de la Red de Profesionales de Turismo del Ecuador.

\section{Resultados}

Los datos de la presente investigación corresponden al criterio profesional y técnico de la Red de Profesionales en Turismo del Ecuador REDPROTUR que arrojaron los siguientes resultados:

Figura 1: ¿Cree usted que en la asignatura de Educación Física que se imparte en las Universidades del país, se aprenden las bases físicas, técnicas, tácticas, psicológicas y teóricas necesarias para desempeñarse en el medio laboral turístico? 


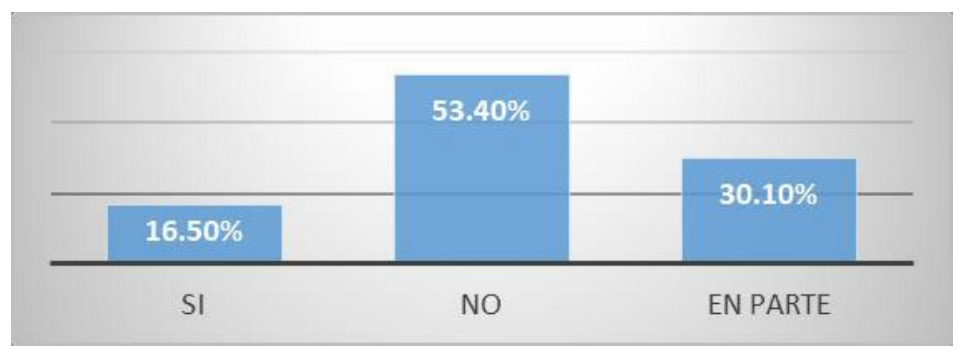

Fuente: Toledo M, Ecuador 2019

El 53,4\% de los encuestados de la Red de Profesionales en Turismo del Ecuador manifiestan que en la asignatura de Educación Física que se imparte en las Universidades del país, NO se aprenden las bases físicas, técnicas, tácticas, psicológicas y teóricas necesarias para desempeñarse en el medio laboral turístico, un $30,1 \%$ opinan que en parte y un $16,5 \%$ piensa que el si se aprenden las bases necesarias para el desempeño laboral turístico.

Figura 2: De lo que usted aprendió en la asignatura de Educación Física en la Universidad ¿Qué tanto le ha servido para su desempeño profesional en el campo turístico?

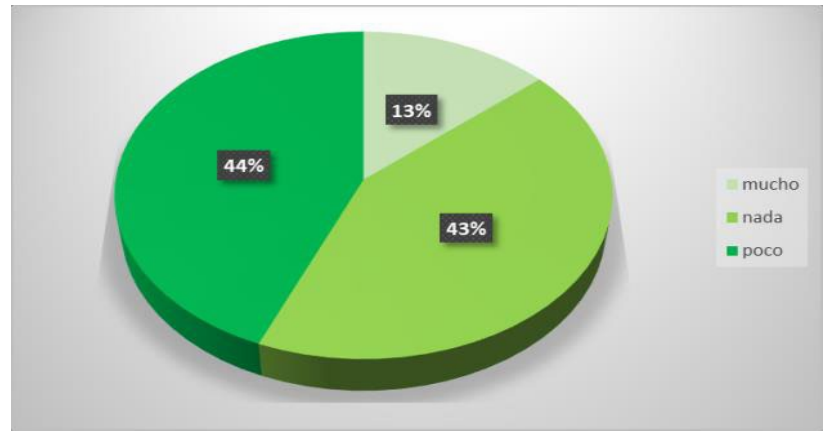

Fuente: Toledo M, Ecuador 2019

Este gráfico nos indica que el $44 \%$ de los encuestados opina que de los conocimientos adquiridos en la asignatura de Educación Física poco le ha servido, un 43\% dice que nada y un $13 \%$ manifiesta que mucho de lo aprendido le servido para su desempeño profesional en el campo turístico.

Figura 3: Según su criterio ¿Qué contenidos y técnicas se deberían desarrollar en la asignatura de Educación Física para desempeñarse como profesionales en el área turística? 


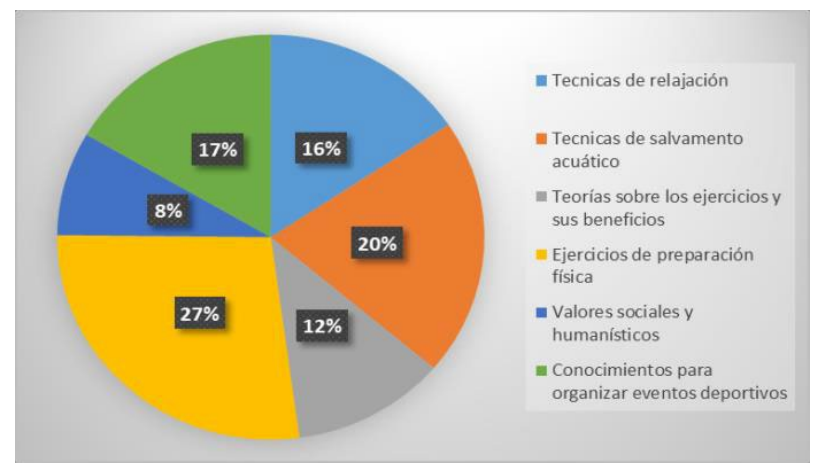

Fuente: Toledo M, Ecuador 2019

El criterio respecto a los contenidos y técnicas se deberían desarrollar en la asignatura de Educación Física para desempeñarse como profesionales en el área turística mayoritariamente recae en los ejercicios de preparación física con un 27\%, seguidos por las técnicas de salvamento acuático con el $20 \%$, posteriormente están los conocimientos para organizar eventos deportivos y técnicas de relajación con un 17\% y $16 \%$ respectivamente, finalmente están las teorías sobre los ejercicios y los valores sociales y humanísticos con el 12\% y el 8\% respectivamente.

Figura 4: ¿Cuántas horas considera usted que debería recibir el estudiante semanalmente en la Universidad para aprender y practicar los conocimientos apropiados en la asignatura de Educación Física?

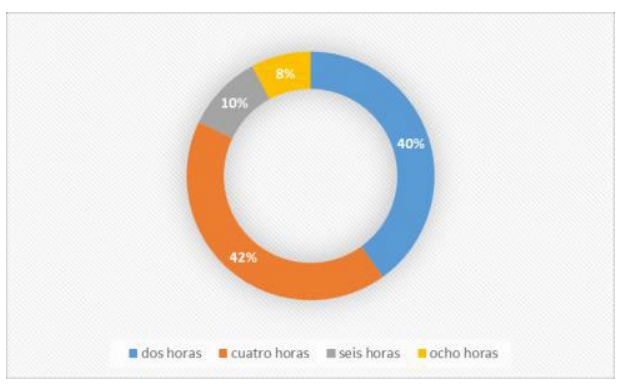

Fuente: Toledo M, Ecuador 2019

Se puede observar que el $42 \%$ y el $40 \%$ de los criterios coinciden en que la carga horaria pertinente para el aprendizaje de los contenidos en la materia de Educación Física debería ser de cuatro a dos horas respectivamente, sin embargo, un porcentaje menor de $10 \%$ y $8 \%$ opinan que se podría prolongar entre seis y ocho horas académicas. 


\section{Discusión}

A partir de los resultados obtenidos en esta investigación se confirma la hipótesis planteada de que el programa actual de Educación Física, que se ejecuta en la mayoría de los pensum académicos de las universidades no aporta con el desarrollo de conocimientos y habilidades que necesita el profesional en el campo laboral turístico enmarcado en el contexto ecuatoriano.

En su mayoría, la Red de Profesionales en Turismo del Ecuador coinciden en que en la asignatura de Educación Física que se imparte en las Universidades del país, no se aprenden las bases físicas, técnicas, tácticas, psicológicas y teóricas que se necesitan para desempeñarse en el medio laboral turístico, y solamente un rango pequeño de encuestados confirman que las bases adquiridas en esta asignatura, si les ha servido en sus labores turísticas.

En cuanto se refiere a los contenidos y técnicas que, según los técnicos, se deberían desarrollar en la asignatura de Educación Física para desempeñarse como profesionales en el área turística mayoritariamente recae en los ejercicios de preparación física, técnicas de salvamento acuático, conocimientos para organizar eventos deportivos y finalmente las técnicas de relajación. Este estudio también revela que esta asignatura no requiere de teoría sino de la práctica, pero una práctica aplicable a las actividades turísticas. Complementariamente a esta pregunta en la encuesta ejecutada se planteó una pregunta tipo abierta cuyo objetivo fue saber cuáles eran las actividades o conocimientos que desde la experticia y verdadera necesidad de los turismólogos se necesita aprender para desarrollar su trabajo en el campo y las respuestas más coincidentes fueron las siguientes:

- Adaptación, aclimatación y normas básicas de supervivencia.

- Deportes de aventura como trekking, escalada, ascenso y descenso de cascadas y montañas.

- Primeros auxilios y técnicas de rescate en zonas agrestes

- Actividades de animación y recreación turística

- Nutrición y dietética deportiva

- Yoga, danza autóctona y expresión corporal.

Con todos estos aportes y criterios algunos especialistas, profesionales y expertos en turismo opinan que se debería eliminar la asignatura de Educación Física y remplazarla por una materia que se denomine "Preparación Física para la actividad Turística". 
Finalmente, en lo que respecta al número de horas pertinente, la mayoría de los entendidos en turismo concuerdan en que la carga horaria para el aprendizaje de los contenidos en la materia de Educación Física se puede enmarcar en horarios que van de dos a cuatro horas.

Es menester indicar que el criterio emitido por este grupo de profesionales no representa a todos los turismólogos del Ecuador sin embargo es una muestra bastante representativa que aporta con datos que demuestran una clara deficiencia en el sistema educativo superior en este caso en lo que compete a la asignatura de Educación Física, en las carreras de turismo o afines.

\section{Conclusiones}

Es imperante la necesidad de que los organismos responsables de la educación superior en el Ecuador puedan tomar en cuenta este tipo de investigaciones de carácter técnico, y que, en base a estos criterios, puedan trabajar en políticas de reestructuración de las mallas curriculares, programas y contenidos de la asignatura de Educación Física de tal forma que estén direccionados y enfocados a la realidad laboral turística del país. Si los institutos, universidades y escuelas politécnicas en sus proyectos de diseño y rediseño curricular presentados al Consejo de Educación Superior CES toman como base referencial este tipo de investigaciones aplicadas a quienes son protagonistas directos de la actividad turística y que emiten su criterio con conocimiento de experiencia, es posible que los contenidos básicos del meso y micro currículo se construyan y se desarrollen enfocados a la realidad laboral del contexto turístico. En este caso se ha tomado como ejemplo la asignatura de Educación Física sin embargo esta misma dinámica se debe aplicar para todas las materias de una carrera determinada, de esta forma se podrá tener la certeza de que los estudiantes y futuros profesionales tienen una base de formación sólida y coherente con la realidad.

La asignatura de Educación Física debe constituirse en un aporte en la formación profesional del turismo como una parte de la alineación del ser humano que tiende al mejoramiento integral de la mente, cuerpo y espíritu, no siendo suficientes los conocimientos adquiridos en las aulas universitarias, sino que los contenidos deben ser fortalecidos y complementados en la práctica.

Gran parte de las demandas actuales de los servicios turísticos, van dirigidas al turismo de deporte y otras actividades físicas en modalidades pasivas y activas donde la parte física recreativa se convertirse en el gran motivador y aliciente turístico. Los ciudadanos en su tiempo 
de ocio, cada vez más se dedican a desarrollar actividades dinámicas, conformando un gran espacio de actuación e intervención que se definiría como turismo activo. La formación de los estudiantes en las aulas de clase universitarias no debería distar de lo que ya en el campo profesional se establece, los programas o paquetes turísticos que se comercializan en turismo incluyen en su mayoría como un principal componente las actividades físicas recreativas, mayoritariamente desarrolladas en el medio natural, siendo parte del turismo de naturaleza, ecoturismo, turismo rural, turismo de salud, turismo de aventura, entre otros, por lo tanto es claro que la asignatura de Educación Física debe estar a la par en la formación basada en el verdadero contexto turístico.

\section{Agradecimientos}

El reconocimiento y agradecimiento del equipo de investigación, a los integrantes de la Red de Profesionales de Turismo en el Ecuador REDPROTUR quienes con su criterio basado en su experticia aportaron con la información obtenida en este trabajo investigativo, y como no puede ser de otra manera a las autoridades de la Escuela Superior Politécnica de Chimborazo Extensión Norte Amazónica.

\section{Referencias Bibliográficas}

Asamblea del Ecuador, C. (2008). Asamblea Nacional. Quito. Ecuador.

Fresneda, C., Herrera, A., \& Álvarez, H. (2013). La Educación Física universitaria: una actualidad con perspectiva. EFDeportes.com, Revista Digital, 17(178), 1-1. Obtenido de http://www.efdeportes.com/efd178/la-educación-fisica-universitaria-una-actualidad.htm.

Charchabal, D, Aguirre, A, Pérez, M. (2003). Elaboración del modelo integral del proceso docente de educativo de Educación Física para la carrera de Ingeniería en Minas.

Revista digital Obtenido de http://www.efdeportes.com.

González, M., \& Suárez, J. (2012). ORGANIZACIÓN DE EVENTOS DEPORTIVOS Y GESTIÓN DE PROYECTOS: FACTORES, FASES Y ÁREAS. Revista Internacional de Medicina y Ciencias de la Actividad Física y el Deporte, 12(45), 138-169. Obtenido de Http://cdeporte.rediris.es/revista/revista45/artorganizacion209.htm.

Luque, A. (15 de diciembre de 2003). LA EVALUACIÓN DEL MEDIO PARA LA PRÁCTICA DE ACTIVIDADES TURÍSTICO.DEPORTIVAS EN LA NATURALEZA. 131-149. Obtenido de http://revistas.um.es/turismo/article/view/19111. 
Ley del Deporte, L. (2010). Educación Física y Recreación. República del Ecuador, Registro Oficial, 255.

MINTUR. (2016). Boletín de estadísticas turísticas 2011-2015. Informe estadístico, Quito.

MINTUR. (6 de junio de 2017). 2017. Obtenido de www.turismo.gob: http://servicios.turismo.gob.ec/index.php/portfolio/turismo-cifras/20-ofertaturistica/servicios-turisticos $/ 247 \mathrm{p}$.

MINTUR. (2017). Proyecto "Programa Nacional para la excelencia turística 2014-2017". Quito. Recuperado el de://www.turismo.gob.ec/wp-content/uploads/2015/04/DocumentoPrograma-Nacional-para-la-Excelencia-Turistica1.pdf.

MINTUR. (2014). Reglamento de Operación Turística de Aventura. Obtenido de http://www.turismo.gob.ec/wp-content/uploads/2016/04/REGLAMENTO-DETURISMO-DEAVENTURA.pdf.

Montes de Oca, N, \& Machado, E. (2009). El desarrollo de habilidades investigativas en la educación superior: Un acercamiento para su desarrollo. Revista de la Revista de Centro de Estudios de Ciencias de la Educación Universidad de Camagüey. Cuba 29 p.

Zagalaz, L., Moreno, R., \& Chacón, J. (2001). NUEVAS TENDENCIAS EN LA EDUCACIÓN FÍSICA. Contextos Educativos, 4, 263-294. 